Quaestio facti. Revista Internacional sobre Razonamiento Probatorio

Quaestio facti. International Journal on Evidential Legal Reasoning Vol. 1 | 2020 pp. $67-74$

Madrid, 2020

DOI: $10.33115 /$ udg_bib/qf.i0.22326

Marcial Pons Ediciones Jurídicas y Sociales

(C) Giulio Ubertis

ISSN: 2604-6202

Recibido: 09/06/19 | Aceptado: 08/07/19

\title{
QUAESTIO FACTI E QUAESTIO IURIS
}

Giulio Ubertis

Professore ordinario di Diritto processuale penale ed Epistemologia giudiziaria nell'Università Cattolica del Sacro Cuore di Milano giulio.ubertis@unicatt.it

ABSTRACT: L'epistemologia giudiziaria studia i metodi e la validità della conoscenza nella ricostruzione giudiziale del fatto. Quindi, riguarda particolarmente la quaestio facti. Ma non si può ignorare l'indissolubile nesso esistente tra quest'ultima e la quaestio iuris. Anche la competenza di legittimità della Corte di cassazione può fondarsi non su una separazione tra questioni di fatto e questioni di diritto, ma sul divieto di svolgere attività probatoria o sulla limitazione, ad esempio, delle violazioni processuali che può esaminare.

PAROLE CHIAVE: Corte di cassazione; Epistemologia giudiziaria; Quaestio facti; Quaestio iuris.

\section{QUAESTIO FACTI AND QUAESTIO IURIS}

ABSTRACT: Judicial epistemology studies the methods and the validity of knowledge in the judicial reconstruction of the fact. Therefore, it refers particularly to quaestio facti. But the indissoluble relationship between the latter and quaestio iuris cannot be ignored. Also, the competence of legitimacy of the Court of cassation may be based not on a separation of question of fact from question of law, but on a prohibition of proceeding with evidentiary investigation or on a limitation, for example, of the breaches of procedure it may examine.

KEYWORDS: Court of cassation; Judicial epistemology; Quaestio facti; Quaestio iuris.

SOMMARIO: 1 . Il titolo di una rivista.-2. La prospettiva giuridica orienta il giudizio di fatto.3. ... e il giudizio di diritto implica quello di fatto.—4. La competenza dalla Corte di cassazione. 


\section{IL TITOLO DI UNA RIVISTA}

La rivista, sul cui primo numero ho l'onore di presentare questo sintetico contributo, è particolarmente importante e innovativa nel panorama internazionale delle pubblicazioni giuridiche periodiche, perché istituisce espressamente una sede dove unificare le esperienze del mondo europeo continentale, anglosassone e latino-americano (con interessamento anche a quello cinese) in maniera interdisciplinare e in un ambito di ricerche ancora a uno stadio iniziale.

Sebbene, infatti, il tema della prova e del ragionamento giuridico sia stato in ogni epoca e inevitabilmente oggetto di indagine da parte degli studiosi del processo giudiziario (civile, penale o amministrativo che fosse) e degli operatori nelle aule di giustizia, fino a poco tempo fa è mancata la consapevolezza che una disamina adeguata della materia è possibile unicamente con il confluire di diverse competenze: giuridiche certamente, ma pure logiche, filosofiche, psicologiche, sociologiche ed epistemologiche.

Di epistemologia giudiziaria, ad esempio, si è cominciato a parlare nel 1979 in Italia $^{1}$, rilevando l'esigenza di esaminare in modo non settoriale i metodi e la validità della conoscenza conseguita in campo processuale: e ciò può effettuarsi coniugando la tradizionale riflessione tecnico-giuridica con la considerazione del dibattito filosofico-scientifico contemporaneo.

Forse proprio la ripulsa di un'attenzione esclusivamente giuridica all'argomento e invece aperta agli esiti degli sviluppi scientifici, usualmente ritenuti più strettamente connessi a elementi fattuali, ha indotto a scegliere un titolo in cui chiaramente emergesse il riferimento all'attività giudiziale nel suo aspetto della ricostruzione fattuale, sul cui giudizio di verità si fonda l'emanazione di una decisione giusta, «indipendentemente dal criterio giuridico che si impiega per definire e valutare la giustizia della decisione» ${ }^{2}$. In proposito, è significativo che il legame (e quindi il rifiuto di una omologazione) tra verità e giustizia sia normativamente confermato in Italia dalla formula del giuramento prevista per i giudici popolari nei giudizi di assise (art. 30 comma 1 1. 10 aprile 1951 n. 287), dove, scolpendosi i caratteri della sentenza, si prescrive che essa «riesca quale la società deve attenderla: affermazione di verità e di giustizia».

Tuttavia due precisazioni si rendono ineludibili.

La prima concernel'avvertenza che nel processo si accerta la «verità di una proposizione» ${ }^{3}$ ela prova, dunque, non concerne mai un "fatto" e riguarda sempre un "enunciato" ${ }^{4}$ :

1 UBERTIS, 1979: 54 ss.

2 TARUfFo, 1992: 43.

3 Carrara, 1886: 201.

4 Anche per evitare fastidiose ripetizioni, si impiegano sinonimicamente vocaboli quali "enunciato", "proposizione", "asserzione" o "affermazione" (di cui, in ogni caso, manca nella relativa letteratura un utilizzo uniforme), poiché dovrebbe emergere facilmente dal contesto quando ci si riferirà all'espressione linguistica o quando si intenderà richiamarne il contenuto (siffatta distinzione è stata recentemente esemplificata in una monografia di epistemologia giudiziaria da Garbolino, 2014: 20 ss.). 
«si può parlare di 'verifica' solo in relazione a proposizioni»" 5 . L'iniziale affermazione probatoria risulta vera ("provata») e si può pertanto dire di "conoscere un fatto" quando, dopo aver instaurato un confronto con un altro enunciato (quello risultante dall'esperimento probatorio), si riscontra che le due asserzioni coincidono. Appunto perché "per controllare un giudizio ne occorre un altro, a cui commisurare il primo» ${ }^{6}$ o, detto altrimenti, «il controllo delle sue proposizioni protocollari il giudice deve compierlo attraverso il raffronto con altre proposizioni dello stesso tipo» ${ }^{7}$, il processo si celebra all'interno di un universo linguistico.

La seconda verte sulla sottolineatura che la quaestio facti è comunque in un rapporto di unità-distinzione con la quaestio iuris: e questo è l'oggetto del presente scritto.

\section{LA PROSPETTIVA GIURIDICA ORIENTA IL GIUDIZIO DI FATTO}

È invero difficilmente contestabile che esista una «reciproca interdipendenza tra il lavoro diretto alla ricostruzione del fatto (nei suoi profili oggettivi e soggettivi) e quello volto alla qualificazione giuridica di quanto dedotto in giudizio» ${ }^{8}$. Quando infatti si intende esaminare un fatto per valutarne gli effetti giuridici o la corrispondenza a norme di diritto, non è possibile evitare di guardarlo con l'uso del reticolo da esse fornito.

Come è stato da tempo riconosciuto, unicamente un "senso mistico della legge»" combinato con «un'osservazione superficiale ... ha potuto far ritenere ... che fosse possibile distinguere ... un giudizio di fatto, ed uno di diritto ${ }^{10}$ tra loro nettamente separati.

Il «fatto» è determinato selettivamente con una scelta compiuta nell'ottica della sua "giuridicità» e risulta quindi impossibile, pure nel successivo momento della verifica processuale, trascurare il contesto teorico dove era stato anteriormente compreso: non può aversi una situazione in cui il fatto "in quanto tale» si trovi in posizione dicotomica rispetto al diritto "in quanto tale".

L'allegazione di parte non è mai un mero "giudizio di fatto» e parimenti non è tale la decisione del giudice, dove «il résulte que les faits établis ne sont pas du tout 'des faits purs' " ${ }^{11}$, tanto più quando si rammenti che la loro conoscenza è inevitabilmente ottenuta con un'attività disciplinata da regole, specialmente probatorie, che caratterizzano qualunque sistema processuale.

5 Neurath, 1932-1933: 59.

6 Cordero, 1963: 6. Analogamente, per Carnelutti, 1958: 129, «la prova (di un giudizio) non può consistere se non in un giudizio diverso".

7 Denti, 1957: 6-7.

8 UBERTIS, 2015: 71, evitando ulteriori richiami quando si ripropongano considerazioni contenute in tale volume.

9 SatTA, 1962: 799.

10 Benvenuti, 1953: 65.

11 Perelman, 1961: 271. 
Gli attori nei processi civili (ma anche i ricorrenti e il pubblico ministero in quelli amministrativi e penali), quando si rivolgono al giudice, «non prospettano fatti nudi, ma fatti qualificati (non per nulla l'art. 163, n. 3 [rectius: 4], C. Proc. Civ. esige, nell'atto di citazione, gli elementi di diritto accanto alla esposizione dei fatti)» ${ }^{12}$, perché «il fatto nel processo non è mai pensato ed espresso isolatamente in funzione della sua verità meramente fattuale ... ma in essenziale relazione con una norma giuridica» ${ }^{13}$.

Né va ignorato che «l'attività di ricostruzione probatoria influenza l'attività di qualificazione normativa piegandola alle proprie circoscritte possibilità euristiche» ${ }^{14}$, «il fatto non è un dato che viene accertato nella sua pretesa oggettività empirica, ma l'esito di un processo ricostruttivo disciplinato dal diritto in funzione delle sue finalità e dei suoi valori» ${ }^{15}$.

Gli unici fatti pertinenti asseriti in ogni processo sono quelli da cui possano scaturire conseguenze giuridiche ${ }^{16}$, ossia fatti giuridici ${ }^{17}$, non potendosi accogliere l'opinione di $\mathrm{chi}^{18}$ voglia ritenere deducibili nel processo fatti non giuridici (sarebbero quelli corrispondenti ai vari casi della vita, su cui si fondano le ricostruzioni delle parti e del giudice), riconosciuti peraltro indirettamente idonei alla fissazione della res in iudicium deducta.

Occorre ribadire che «il 'fatto' del giurista non è un fatto per così dire grezzo, primordiale, metagiuridico, ma il fatto rappresentato in funzione di un interesse giuridico ${ }^{19}$ e sempre, in quanto giuristi, «noi guardiamo al fatto attraverso la norma» ${ }^{20}$.

\section{3. ... E IL GIUDIZIO DI DIRITTO IMPLICA QUELLO DI FATTO}

D’altra parte, si è evidenziata «una 'essenza relazionale' della fattispecie» ${ }^{21}$, sostenendo che lo stesso "giudizio di diritto implica, in realtà, il giudizio di fatto» ${ }^{22}$.

12 V. ANDRIOLI, 1967: 261.

13 NASI, 1967: 987

14 Carlizzi, 2006: 1195.

15 Vogliotti, 2007: 239.

16 Perelman, $1959: 212$, ha pertanto sostenuto che, per le parti in causa, sono i provvedimenti giurisdizionali «qui leur importent le plus souvent, bien plus que la réalité des faits, qui ne constituent qu'un moyen de fonder les conséquences juridiques qui en découlent».

17 Conso, 1955: 44, ad esempio, definisce «fatto giuridico ogni fatto rilevante per il diritto nel senso che da solo o, per lo meno, insieme ad altri (che saranno, pure essi, fatti giuridici) è produttivo di conseguenze giuridiche».

18 TARUfFo, 1971: 40.

19 Rotondi, 1977: 952.

20 IRTI, 1967: 95.

21 Pisani, 1959: 16, aggiungendo che "per questo la scelta della fattispecie primaria si opera per tentativi, ad ognuno dei quali sottende un problema d'interpretazione, al fine di vagliare comparativamente le possibili conseguenze che scaturiscono dall'adottare come applicabile l'uno o l'altro schema valutativo" (ivi, 17).

22 Carnelutti, 1956: 93. 
Il nesso tra i due giudizi è inevitabile dato che la tensione alla giustizia sottostante a tutto l'ordinamento si rivela altresì nell'attività interpretativa volta all'individuazione della norma applicabile. Pure l'analisi sub specie iuris postula «l'uso degli strumenti propri del discorso valutativo: si pensi al ragionamento analogico, mediante il quale si perviene all'identificazione dell'identità di ratio tra il caso non regolato e quello esplicitamente previsto" ${ }^{23}$.

Similmente al giudizio concernente la quaestio facti, che non può concernere un "puro fatto" per la sua ineludibile collocazione interna alle coordinate giuridiche, il "giudizio di diritto" non concerne una regola astratta e slegata dall'esperienza umana, ma una norma destinata a trovare attuazione nel mondo concreto, dove è necessaria e imprescindibile la correlazione tra il precetto come scelto e interpretato e il "fatto" come ricostruito: «le norme sono interpretate in funzione dei fatti e questi ricostruiti in funzione di quelle» ${ }^{24}$.

Le componenti della coppia rappresentata da fatto e diritto, quaestio facti e qaestio iuris, giudizio di fatto e giudizio di diritto costituiscono costruzioni intellettuali tra loro inscindibili, dove pensiero e azione sono intimamente fusi ${ }^{25}$ e il cui carattere distintivo è fondamentalmente metodologico. Esse, "progressivamente e reciprocamente determinantisi ${ }^{26}$, sono generalmente oggetto di esame disgiunto soltanto per risolvere con un'esposizione ordinata i differenti problemi che, in diversi momenti, possono doversi affrontare in ambito processuale.

Si tratta di una distinzione «attuata all'interno di un contesto unitario che, senza tramutarla in identità dei termini, consente di riconoscere questi ultimi non come qualitativamente separati ma come portatori di una diversa proporzione tra gli atteggiamenti, comunque presenti, della constatazione e della valutazione giuridica» ${ }^{27}$.

\section{LA COMPETENZA DELLA CORTE DI CASSAZIONE}

Disconoscendo le critiche risalenti almeno fino al secolo XIX ${ }^{28}$, spesso viene affermato che sarebbe normalmente «bandito dal giudizio di cassazione il giudizio di fatto» ${ }^{29}$; in questo modo, non si pone attenzione al concreto operare della Corte di cassazione $^{30}$, la cui disamina contribuisce a smascherare tali astratte teorizzazioni.

23 Cordero, 1961: 883.

24 Ferrua, 2017: 41.

25 Perelman, 1961: 278.

26 PASTORE, 1996: 58.

27 UBERTIS, 1979: 76.

28 TARUfFO, 1982: 47, ricorda quelle formulate quando, al tempo dell'unificazione legislativa successiva all'unità d'Italia, si confrontarono i fautori del sistema della Cassazione e i sostenitori di quello della terza istanza.

29 Tonini, 2018: 955.

30 Se «il magistrato del 'controllo' ... si persuade o ha la sensazione di trovarsi di fronte ad una sentenza errata, ingiusta sul terreno del giudizio di fatto, ... trova il suo sfogo nell'annullamento, per 
L'asserita dicotomia tra il «fatto» e il «diritto», infatti, non è riconducibile a un ipostatizzato "dato oggettivo" precedente all'interpretazione ${ }^{31}$ ed è funzionale a un (elastico) canone di (auto)determinazione della competenza ${ }^{32}$ : senza ignorare che il medesimo "giudizio di diritto" va reputato "di fatto" nella misura in cui si consideri che esso concerne un "fatto" costituito da una norma giuridica ${ }^{33}$.

D'altra parte, è stato da tempo rilevato che la Corte di cassazione, nel momento in cui afferma che la questione sottopostale è "di fatto» e che pertanto appartiene alla competenza del giudice di grado inferiore, sostanzialmente indica agli interessati ritiene di affidare a questo il compito della sua qualificazione giuridica ${ }^{34}$. il «giudizio di fatto" che la Cassazione rinuncia a esprimere è comunque impregnato di diritto anche perché individuato come tale da un "giudizio di diritto» che lo estromette dalla propria sfera di applicazione.

Quando non le sembra adeguata la decisione che sta esaminando, la Corte di cassazione può sempre operare una "conversione» della questione di fatto in questione di diritto, inserendola all'ambito della propria competenza. Secondo un ormai celebre esempio ${ }^{35}$, l'esegesi della disposizione secondo cui l'usufrutto cessa con la «morte naturale» dell'usufruttuario non pare dare adito a dubbi. Il quadro, tuttavia, muterebbe qualora tale soggetto venisse a subire un procedimento scientifico che permettesse di conservarlo indefinitamente in stato di ibernazione. La stessa nozione di «morte naturale», che fino poco prima rappresentava una indiscussa "questione di fatto», diventerebbe agevolmente una «questione di diritto» idonea a provocare al riguardo un provvedimento della Corte di cassazione.

Poiché la separazione tra fatto e diritto non è quindi in grado di fondare la ripartizione della competenza tra la Corte di cassazione e i giudici di grado inferiore, occorre quindi definire un criterio di tipo quantitativo ed estrinseco rispetto alle forme del ragionamento sul quale viene eseguito il controllo.

In questa prospettiva di soluzione, conviene rammentare un peculiare significato attribuito al vocabolo «merito", «sovente usato per indicare la questio facti dibattuta nel processo e le correlative indagini probatorie» ${ }^{36}$ e spesso adoperato per individuare

mancanza o contraddittorietà della motivazione che schiude la via alla riparazione della (presunta) denegata giustizia» (Zerboglio, 1943: 738-739).

31 AsCARELLI, 1961: 128.

32 FORIERs, 1961: 77.

In particolare, nel diritto processuale amministrativo «le problème de la distinction du fait et du droit dévient celui de la distinction entre légalité et opportunité» (RIvero, 1961:132) e «i confini della legittimità sono talmente elastici ... che non sono essi a definire l'estensione del sindacato giurisdizionale quanto, piuttosto, è un costante orientamento giurisprudenziale a determinare, per qualche aspetto, il contenuto della legittimità medesima» (Levi, 1967: 154).

33 Sinteticamente, si è evidenziato che, «come il cosiddetto giudizio di fatto è l'accertamento del fatto del privato, così il cosiddetto giudizio di diritto è l'accertamento del fatto del legislatore» (CALOGERO, 1937: 138).

34 Perelman, 1961: 277.

35 Perelman, 1961: 275, dove il richiamo era effettuato all'art. 617 c.c. belga.

36 Cordero, 1964: 580 (corsivo nostro). 
la competenza degli organi giurisdizionali diversi dalla Cassazione, alla quale viene riservata la denominazione di "giudice di legittimità» ${ }^{37}$.

Tale qualifica può giustificarsi con la circostanza secondo cui essa, essendole vietato di svolgere istruzione probatoria, deve provvedere ad «accertare che il fatto sia stato compreso secondo quello che è il suo significato valido per tutti» ${ }^{38}$, effettuando "non già una ricostruzione e gestis, ma un rilievo ex actis» ${ }^{39}$. In altre parole, la sua funzione è costituita dal "verificare, mediante un controllo semantico ex actis e senza poter dividere nettamente il 'fatto' dal 'diritto', se il 'fatto giuridico' dedotto in giudizio sia stato, alla fine, reso comunicabile e comprensibile dal giudice di merito» ${ }^{40}$. E l'area di intervento della Cassazione potrà avere un'estensione ancora più ridotta, circoscrivendo ciò che può sottoporsi al proprio vaglio (è quanto accade nell'ordinamento italiano, dove la violazione delle norme processuali penali è motivo di ricorso per cassazione soltanto qualora siano «stabilite a pena di nullità, di inutilizzabilità, di inammissibilità o di decadenza»: art. 606 comma 1 lett. c c.p.p.). Ma dovrà comunque accertarsi che la conoscenza del «fatto» cui è dedicata la sua disamina sia stata ottenuta attraverso la sua adeguata inserzione in quel modello concettuale comune ai consociati, che, pure nello spazio costituzionalmente tutelato delle diverse opzioni ideologico-culturali, è determinato dall'insieme delle norme giuridiche.

Restano i timori che la Cassazione possa così restringere la libertà di giudiziodegli organi giurisdizionali di grado inferiore. Siffatte preoccupazioni, tuttavia, non vengono certo eliminate con l'impiego di infondate teorizzazioni (come quella della netta divisione tra questio facti e quaestio iuris), ma da idonee riforme dell'ordinamento giudiziario: tale sarebbe la previsione di «un sistema in cui le funzioni di legittimità attribuite alla Corte siano affidate alla magistratura nella sua totalità ... Il conferimento temporaneo delle funzioni di legittimità permetterebbe uno scambio dialettico fra dette funzioni e le funzioni di merito e, per questa via, consentirebbe la sintesi dei vari orientamenti giurisprudenziali» ${ }^{41}$.

\section{BIBLIOGRAFIA}

Andrioli, V., 1967: «Prova (diritto processuale civile)», in Novissimo digesto italiano, XIV. Torino: Utet, 260-300.

Ascarelli, T., 1961: "Le fait et le droit devant la Cour de cassation italienne», in Le fait et le droit. Etudes de logique juridique. Bruxelles: Bruylant, 113-129.

BARGIS, M., 2018: «Impugnazioni», in Compendio di procedura penale, a cura di M. Bargis. Milano: Wolters Kluwer - Cedam, 855-1025.

Benvenuti, F., 1953: Listruzione nel processo amministrativo. Padova: Cedam.

\footnotetext{
37 Cfr., per tutti, Bargis, 2018: 942.

38 NASI, 1967: 986.

39 Calogero, 1937: 183.

40 UBERTIS, 1979: 79-80.

41 Siracusano, 1974: 1246.
} 
Calogero, G., 1937: La logica del giudice e il suo controllo in Cassazione, ristampa 1964. Padova: Cedam.

Carlizzi, G., 2006: «Ragionamento giudiziario e complessità diacronica del circolo ermeneutico», in Cassazione penale: 1184-1204.

Carnelutti, F., 1956: «Nuove riflessioni sul giudizio giuridico», in Rivista di diritto processuale, I: 81106.

- 1958: Diritto e processo. Napoli: Morano.

Carrara, F., 1886: Programma del corso di diritto criminale. Parte generale, III. Prato: Giachetti.

Conso, G., 1955: I fatti giuridici processuali penali. Perfezione ed efficacia. Milano: Giuffrè.

Cordero, F., 1961: "Giudizio», in Novissimo digesto italiano, VII. Torino: Utet, 881-886.

- 1963: «Il procedimento probatorio», in ID., Tre studi sulle prove penali. Milano: Giuffrè, 3-144.

- 1964: «Merito (diritto processuale», in Novissimo digesto italiano, X. Torino: Utet, 578-581.

Denti, V., 1957, La verificazione delle prove documentali. Torino: Utet.

Ferrua, P., 2017: La prova nel processo penale, I, Struttura e procedimento. Torino: Giappichelli.

Foriers, P., 1961: «La distinction du fait et du droit devant la Cour de cassation de Belgique», in Le fait et le droit. Etudes de logique juridique. Bruxelles: Bruylant, 51-77.

Garbolino, P., 2014: Probabilità e logica della prova. Milano: Giuffrè.

IRTI, N., 1967: «Rilevanza giuridica», in Jus: 55-108.

LeVI, F., 1967: L'attività conoscitiva della pubblica amministrazione. Torino: Giappichelli.

NASI, A., 1967: "Fatto (giudizio di)», in Enciclopedia del diritto, XVI. Milano: Giuffrè, 967-988.

Neurath, O., 1932-1933: «Proposizioni protocollari», trad. it., in ID., Sociologia e neopositivismo, 1968. Roma: Ubaldini, 54-64.

Pastore, B., 1996: Giudizio, prova, ragion pratica. Un approccio ermeneutico. Milano: Giuffrè.

Perelman, Ch., 1959: «La spécificité de la preuve juridique», in ID., Justice et raison, 1963. Bruxelles: Presses Universitaires de Bruxelles, 206-217.

— 1961: «La distinction du fait et du droit. Le point de vue du logicien», in Le fait et le droit. Etudes de logique juridique. Bruxelles: Bruylant, 269-278.

Pisani, M., 1959: La tutela penale delle prove formate nel processo. Milano: Giuffrè.

Rivero, J., 1961: «La distinction du droit et du fait dans la jurisprudence du Conseil d'Etat français», in Le fait et le droit. Etudes de logique juridique. Bruxelles: Bruylant, 130-148.

Rotondi, M., 1977: "Considerazioni in "fatto" e in "diritto" , in Rivista trimestrale di diritto e procedura civile: 949-968.

SATTA, S., 1962, "Corte di cassazione: c) diritto processuale civile», in Enciclopedia del diritto, X. Milano: Giuffrè, 797-829.

Siracusano, D., 1974: "Certezza del diritto e Cassazione penale», in Studi in memoria di O. Condorelli, III. Milano: Giuffrè, 1243-1247.

TARuffo, M., 1971: «Note in tema di giudizio di fatto», in Rivista di diritto civile, I: 33-51.

— 1982: "Cassazione e revisione: un problema nella storia delle istituzioni giudiziarie», in Materiali per una storia della cultura giuridica: 41-60.

- 1992, La prova dei fatti giuridici. Nozioni generali, in Trattato di diritto civile e commerciale, già diretto da A. Cicu - F. Messineo e continuato da L. Mengoni, III, 2, 1. Milano: Giuffrè.

Tonini, P., 2018: Manuale di procedura penale. Milano: Giuffrè.

Ubertis, G., 1979: Fatto e valore nel sistema probatorio penale. Milano: Giuffrè.

— 2015: Profili di epistemologia giudiziaria. Milano: Giuffrè.

Vogliotti, M., Tra fatto e diritto. Oltre la modernità giuridica. Torino: Giappichelli.

Zerboglio, A., 1943: "Appunti sulla motivazione della sentenza penale», in Studi in memoria di F. Ferrara, II. Milano: Giuffrè, 729-741. 